\title{
Modeling flexibility using artificial neural networks
}

\author{
Kevin Förderer ${ }^{1 *}$, Mischa Ahrens ${ }^{2}$, Kaibin Bao $^{2}$ (D), Ingo Mauser ${ }^{2}$ (D) and Hartmut Schmeck ${ }^{1,2}$ (D) \\ From The 7th DACH+ Conference on Energy Informatics \\ Oldenburg, Germany. 11-12 October 2018
}

\footnotetext{
*Correspondence: foerderer@fzi.de

${ }^{1}$ FZI Research Center for

Information Technology,

Haid-und-Neu-Str. 10-14, 76131

Karlsruhe, Germany

Full list of author information is

available at the end of the article
}

\begin{abstract}
The flexibility of distributed energy resources (DERs) can be modeled in various ways. Each model that can be used for creating feasible load profiles of a DER represents a potential model for the flexibility of that particular DER. Based on previous work, this paper presents generalized patterns for exploiting such models. Subsequently, the idea of using artificial neural networks in such patterns is evaluated. We studied different types and topologies of ANNs for the presented realization patterns and multiple device configurations, achieving a remarkably precise representation of the given devices in most of the cases. Overall, there was no single best ANN topology. Instead, a suitable individual topology had to be found for every pattern and device configuration. In addition to the best performing ANNs for each pattern and configuration that is presented in this paper all data from our experiments is published online. The paper is concluded with an evaluation of a classification based pattern using data of a real combined heat and power plant in a smart building.
\end{abstract}

Keywords: Smart Grid, Modeling, Flexibility, Distributed energy resources, Demand side management, Machine learning

\section{Introduction}

Traditionally, electricity has almost exclusively been produced in large power plants connected to electricity transmission grids. The growing share of distributed energy resources (DERs) connected to distribution grids makes reliable grid operation increasingly challenging. Since solar and wind energy are volatile in nature, generation by DERs that use these energy sources is intermittent. To limit the extent of necessary grid and energy storage expansion, the exploitation of the already existing flexibility of DERs like battery energy storage systems (BESSs) and combined heat and power (CHP) plants is essential. Aside from conventional measures of demand response (DR), newer approaches to achieve a comprehensive demand side management (DSM) (Palensky and Dietrich 2011) have been proposed, including hierarchical (Molderink 2011; Anders et al. 2014; Toersche et al. 2015), distributed (Callaway and Hiskens 2011; Hinrichs and Sonnenschein 2017), decentralized (Bremer and Lehnhoff 2017; Rohbogner et al. 2014) and cellular systems (Mauser et al. 2017b; Waffenschmidt 2017). A common necessity for all these approaches is the need to at least model and often communicate flexibility.

(c) The Author(s). 2018 Open Access This article is distributed under the terms of the Creative Commons Attribution 4.0 International License (http://creativecommons.org/licenses/by/4.0/), which permits unrestricted use, distribution, and reproduction in any medium, provided you give appropriate credit to the original author(s) and the source, provide a link to the Creative Commons license, and indicate if changes were made. 
The flexibility of a particular DER or an aggregate of multiple DERs can be described as the set of all feasible load profiles for a given time frame. Feasible in the context of DER load profiles refers to load profiles that can be realized based on the current state while providing all necessary services (Mauser et al. 2017a). In this paper we pick up the idea of representing and communicating flexibility with artificial neural networks (ANNs) presented in (Förderer et al. 2018): A single ANN implicitly learns a flexibility model for one or multiple aggregated DERs using generated or measured load profiles and state data relating to the corresponding DERs as training data. It is important to note that a single (measured) load profile does generally give only few clues about the available flexibility. In order to derive an adequate description of the flexibility in a given state, a sufficient number of measured load profiles with comparable initial states is required. Since the ANNs can be trained to consider these initial states, they should be able to deduce the actual flexibility. The ANNs are trained locally and then transmitted to third parties to offer flexibility information and act as surrogate models. Depending on the chosen training pattern, such an ANN could, e.g., evaluate if a given load profile is feasible for a particular DER. This approach enables the abstraction and communication of distributed flexibility regardless of the type, configurations and sizes of the considered DERs. Since a trained ANN may factor in the current state of the corresponding DERs, it is sufficient to only communicate states rather than a complete model once an ANN is known.

This paper aspires to evaluate the idea of using ANNs as surrogate models for flexibility by testing the effectiveness of different ANN types and topologies in conjunction with the patterns presented in (Förderer et al. 2018). By explaining the particularly good or bad results achieved for a given pattern and DER configuration, we aid future research in designing better ANNs that represent energy flexibility. An additional evaluation is conducted using real-world CHP data for one of these patterns.

\section{Related work}

As mentioned before, this paper is based on the ideas outlined in (Förderer et al. 2018). Due to the variety of possible applications for the concept and patterns, the presented results are related to a multitude of previous publications. In this section we give a brief overview of findings motivating the concept and point out important distinctions from other related work.

Regardless of using direct or indirect mechanisms for controlling DERs (Mauser et al. 2017a), it is necessary to employ some sort of model to determine flexibility. For example, customers may respond diversely to time-of-use tariffs (Faruqui and George 2005; Faruqui and Sergici 2010) and even these responses are heavily influenced by local parameters (Jargstorf et al. 2015). Hence detailed models and information are needed.

Recently, machine learning has been shown to be beneficial in energy applications like power system monitoring (Malbasa et al. 2017) and non-intrusive load monitoring (Batra et al. 2014). Artificial neural networks, in particular, have been used for diverse tasks including forecasting of consumption (Rodrigues et al. 2014), solar power (Abuella and Chowdhury 2015), prices (Severini et al. 2015), as well as estimating the duration a heating device is able to provide a requested change in power (MacDougall et al. 2016). One of the most recent applications of ANNs is the automated operation of DERs (Santo et al. 2018). Those applications have in common that the ANN is trained to perform a certain task in the local energy management of a DER, building, facility, virtual power plant, or the like. 
In contrast to these, in the concept presented in (Förderer et al. 2018) and evaluated in this paper the ANN is generated locally but used externally by a third party.

Regarding the concept and the patterns evaluated in this paper, support vector data description (SVDD) (Bremer et al. 2011; Bremer and Sonnenschein 2013; Nieße et al. 2016; Bremer and Lehnhoff 2017) and the cascade classification model (Neugebauer et al. 2015; Neugebauer et al. 2016; Neugebauer et al. 2017) are the most relevant related approaches. SVDD can be used to decide whether a load profile is feasible for a particular DER or an aggregate of DERs or not. Additionally, SVDD (in theory) allows for repairing infeasible load profiles. An important distinction to the models used in this paper is that the SVDD model must be generated each time the flexibility is exchanged. The cascade classification approach is an example for an application of the classification pattern discussed in the next section.

\section{Approach}

Someone who seeks to generate suitable signals, i.e. incentives or commands, in order to influence generation and consumption generally bases their decisions on descriptions of the available flexibility. These descriptions are given by one or multiple models for the flexibility provided by DERs. Since flexibility can be seen as a set of feasible load profiles, the basic function of a model for flexibility is to enable an operator to find suitable profiles. The set of feasible load profiles is often modeled by specifying the definitive constraints. However, any other model allowing the generation of feasible load profiles, e.g. a simple enumeration of load profiles, can pose as a model for the flexibility of DERs.

\section{Realization patterns for modeling flexibility}

The patterns outlined in this section are a generalization of the five usage patterns for ANN-encoded abstracted flexibility presented in (Förderer et al. 2018). We omit pattern $\boldsymbol{E}$ which allows the optimization and communication of load changes rather than absolute load profiles, since it is basically an extension of the other patterns. Each pattern corresponds to pattern specific (surrogate) models. Given one of these pattern specific models, the pattern can be used to generate a set of feasible load profiles. Thereby, the pattern specific model acts as a model for the flexibility of DERs. In the following, we refer to all kinds of third parties performing DSM, e.g. electric utilities (Gellings 1985) or dedicated regional EMSs (Kochanneck et al. 2015), with the term demand side manager (DSMgr). For simplicity, we use the term building when referring to a provider of flexibility. Nevertheless, the concept is applicable to any kind of energy system, such as a single household, a multi-family building, a commercial or industrial property, or a virtual power plant aggregating multiple DERs. Since there are many more possibilities to achieve the goal of generating feasible load profiles, the list of patterns is not exhaustive.

\section{Pattern A: load profile classification}

In the classification pattern the pattern specific model is a classifier. The DSMgr generates load profiles either randomly or according to a given algorithm and validates them, i.e., classifies whether the load profiles are likely to be feasible. By memorizing the discovered feasible profiles, a set of feasible load profiles is generated. This set is used for determining the best load profiles in terms of the DSMgr's goals and the resulting profiles are transmitted to the respective building's EMS. 


\section{Pattern B: price-based load profile forecasting}

The price-based forecasting pattern employs a model that forecasts a load profile for a given arbitrary price signal. By applying the model to various price signals, again, a set of load profiles is generated. After determining the optimal profiles, the corresponding price signal is sent to each building.

\section{Pattern C: load profile generation}

In the generation pattern, the models are used to generate valid load profiles from arbitrary representations. An intuitive type of representation is, for example, a control sequence specifying how a DER operates. By processing the commands in the control sequence the model can generate a load profile. Another type of (latent) representation, usually used in Generative Adversarial Networks, is a vector of random variables. Given a model, the DSMgr creates representations either randomly or according to an algorithm and generates a feasible load profile from each representation. In this pattern, like in pattern $\boldsymbol{A}$, load profiles are selected and transmitted.

\section{Pattern D: load profile validation and repair}

The validation and repair pattern is based on models that allow transforming infeasible load profiles into feasible profiles. Already feasible profiles either remain unchanged when the model is applied or are filtered beforehand, e.g. through pattern $A$. In addition to allowing the generation of feasible load profiles, the validation and repair pattern can simplify the directed search for feasible load profiles if the transformed, i.e. repaired, load profile is similar to the profile given to the model. Again, the target is the identification and communication of desired load profiles.

\section{Communication}

In order to utilize the models for flexibility they need to be available to the DSMgr. Based on the previous section and again (Förderer et al. 2018), this section discusses the transmission and general utilization of the models. For this purpose, quantities are distinguished between parameters and variables. More precisely, parameters are constants inherent to an energy system and variables reflect the current state of the system. A BESS, for instance, can be modeled with the parameter 'storage capacity' that remains fixed and the variable 'state of charge'. Regarding the communication, the model and its parameters only need to be transmitted once. Variables, on the other hand, reflect the current state of the system and thus have to be updated repeatedly.

In detail, initially and whenever the model and/or parameters are outdated, new models are generated and parameters are determined by the EMS of the building and transmitted to the DSMgr's EMS (see Fig. 1):

1. Generate a model and determine the parameters in the local EMS of the building according to the particular usage pattern (cf. patterns $\boldsymbol{A}$ to $\boldsymbol{D}$ )

2. Transmit the model and parameters to the DSMgr

3. Store the received information in the EMS of the DSMgr for later usage

The EMS of the DSMgr may already know suitable (predefined) models simplifying the process to determining only the parameters and transmitting them. 


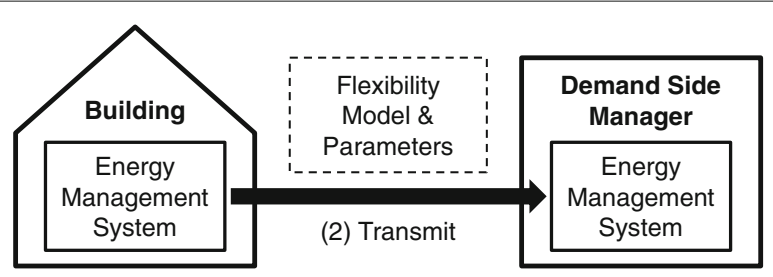

(1) Train

(3) Store

Fig. 1 Model and parameters representing the flexibility of one or multiple DERs in a building are communicated to the demand side manager (cf. (Förderer et al. 2018))

Figure 2 depicts the process of generating feasible load profiles in order to determine a signal for influencing the behavior of the building. Periodically or on demand, the EMS of the building sends the variables relevant to the applied pattern to the DSMgr's EMS. The EMS of the DSMgr then uses the model and parameters it has previously stored, the pattern dependent variables and additional pattern specific inputs (cf. patterns $\boldsymbol{A}$ to $\boldsymbol{D}$ ) to find the optimal load profile and thereby determine the signal to be sent to the EMS of the building.

\section{Concept of ANN-encoded flexibility}

In this paper we evaluate the concept of ANN-encoded abstracted flexibility presented in (Förderer et al. 2018). Therefore, the models for applying the patterns are all based on ANNs. The ANNs represent one or multiple DERs and act as surrogate models. We assume that the variables provided by the building contain the current state of the DERs that are represented by the ANN. The ANN input is then the current state and some pattern specific data (see above) that needs to be generated by the EMS of the DSMgr. Table 1 provides a summary of the pattern specific input and output of the ANNs.

\section{Methodology and models}

Although the patterns presented in this paper all serve the same purpose, which is to enable the DSMgr to exploit the flexibility of DERs by deriving load profiles, they are very different in terms of ANN input and output. Therefore, we have no single source of data suitable for each pattern. The data used for training, testing, and evaluating the ANNs is generated using random load profiles, simulation models, and optimization models. All models are implemented in Python 3.5.2 using the SimPy 3.0.10 simulation framework and Pyomo 5.3 with Gurobi 7.5.2 to solve the mixed integer linear programs (MILPs). Table 2 provides a summary of how the data for each pattern is generated.

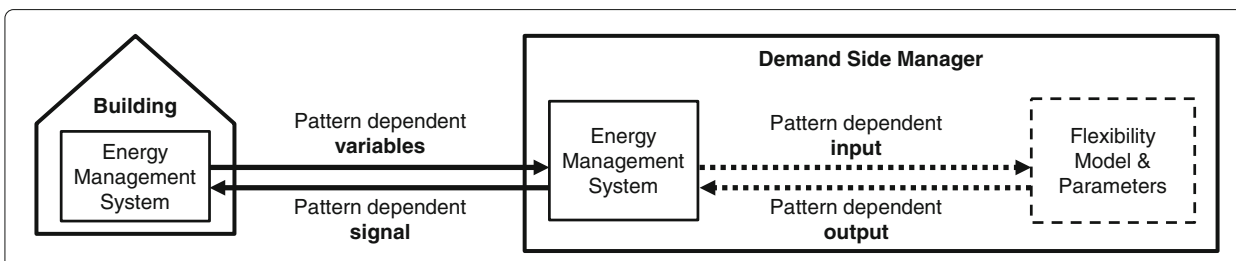

Fig. 2 Based on regularly transmitted variables, e.g. the state of a DER, the demand side manager generates and communicates a signal for influencing the buildings demand. The signal is determined using the models transmitted earlier and the respective patterns (cf. (Förderer et al. 2018)) 
Table 1 Summary of the data given to and generated by the ANNs

\begin{tabular}{llll}
\hline Pattern & Description & ANN Input & ANN Output \\
\hline A & Classification & Current state, generated load profile & Validity of load profile \\
B & Forecasting & Current state, generated price signal & Expected load profile \\
C & Generation & $\begin{array}{l}\text { Current state, generated load profile } \\
\text { representation }\end{array}$ & Expected load profile \\
D & Validation (1) \& repair (2) & Current state, generated load profile & $\begin{array}{l}\text { (1) Validity of load profile } \\
\text { (2) Expected load profile }\end{array}$ \\
\hline
\end{tabular}

Since the simulation models presented in this section recreate the behavior of actual DERs and a single invalid action already leads to an infeasible load profile, the infeasible profiles generated from these models tend to be close to feasible profiles. To create load profiles that are spread evenly throughout the space of all (feasible and infeasible) load profiles, we generate truly random profiles by drawing independent random values for each time slot. The resulting power profiles have a length of $24 \mathrm{~h}$ and a resolution of $5 \mathrm{~min}$. Similarly, all simulation and optimization models generate profiles of the same length and resolution.

For the evaluation of the concept, we consider a household of four persons in three different DER equipment configurations:

1. Battery energy storage system (BESS)

2. Combined heat and power plant (CHP plant)

3. Combination of BESS and CHP plant

Each configuration is different in terms of constraints and the complexity of determining the set of feasible load profiles: While the BESS is allowed to operate freely within the boundaries of its technical restrictions, the CHP plant's operation is limited by the temperature limits of the connected hot water tank and the household's heat demand. Hence, to determine the flexibility of the CHP plant, it is necessary to infer changes to the temperature of the hot water tank and to determine whether the thermal demand can be satisfied or not. The third configuration is used to evaluate the aggregation of multiple heterogeneous DERs. Electric and thermal demands in winter, summer, and intermediate seasons, respectively, are determined from the average of 60 simulations of identical households on a weekday using the CREST Demand Model (McKenna and Thomson 2016). The seasons represent varying consumption patterns and are encoded as three binary variables in the state vector the ANN receives. More detailed information on parameters is given in Table 3.

For the evaluation, the model parameters of the DERs are scaled to not exceed $\pm 1 \mathrm{~kW}$ and the energy demands as well as the hot water tank capacity are scaled accordingly, i.e., divided by 2.75 . In practice, this scaling factor (if applied) would be given to the DSMgr

Table 2 Data sources for each pattern

\begin{tabular}{|c|c|}
\hline Pattern & Data Source \\
\hline $\bar{A}$ & Simulation Model $1^{\mathrm{a}}$ and random load profiles ${ }^{\mathrm{a}}$ \\
\hline B & Optimization with objective (2) \\
\hline C & Simulation Model 2 \\
\hline D & Simulation Model $1^{a}$ and random load profiles ${ }^{a}$ \\
\hline
\end{tabular}


Table 3 Overview of model parameters

\begin{tabular}{lll}
\hline & Description & Detail \\
\hline Electrical demand & Winter & $15.7 \mathrm{kWh} /$ day \\
& Intermediate & $13.9 \mathrm{kWh} /$ day \\
Thermal demand & Summer & $12.5 \mathrm{kWh} /$ day \\
& Winter & $59.9 \mathrm{kWh} /$ day \\
& Intermediate & $46.9 \mathrm{kWh} /$ day \\
BESS & Summer & $18.6 \mathrm{kWh} /$ day \\
& Power & {$[-2.75,2.75] \mathrm{kW}$} \\
& Usable capacity & $2.75 \mathrm{kWh}$ \\
CHP & Efficiency $\eta_{B}$ & 0.92 \\
Hot water tank & Power el. & $0 \mathrm{~kW}$ or $2.75 \mathrm{~kW}$ \\
& Power thermal & $0 \mathrm{~kW}$ or $6.875 \mathrm{~kW}$ \\
& Capacity & $\sim 17.45 \mathrm{kWh}$ \\
& 750 L at $60^{\circ}-80^{\circ} \mathrm{C} \& 20^{\circ} \mathrm{C}$ environment & \\
& Efficiency is border of classes B \& C (European Union 2013$)$ \\
\hline
\end{tabular}

alongside the ANNs. The initial states of charge of the BESS and hot water tank range from 0 to $100 \%$ in steps of $10 \%$. Electricity time-of-use tariffs, which are used in pattern $\boldsymbol{B}$, divide every day into six time slots starting at 06:00, 12:00, 13:00, 17:00, 19:00, and 22:00. Ensuring an average price of $30 \mathrm{cents} / \mathrm{kWh}$ throughout the day, the price for every slot is set to either 24,30 , or 36 cents/kWh which leads to 33 possible combinations per day.

\section{Optimization models}

Multiple MILPs are employed to evaluate the feasibility of load profiles, to repair infeasible profiles, and to find the profile featuring the lowest cost. Evaluation and repair are achieved by minimizing the distance to the target load profile. To measure the distance, we use the Chebyshev distance $L_{\infty}$, i.e., the maximum absolute difference. The objective is:

$$
\min _{p_{\text {Draw }}, p_{\text {FeedIn }}} \max _{k}\left|p_{\text {Draw }, k}-p_{\text {FeedIn }, k}-p_{\text {Target }, k}\right|
$$

with $p_{\text {Draw, }}$ and $p_{\text {FeedIn, } k}$ being the (average) power drawn from or fed into the grid during time slot $k$. In every slot $k$, only one of these two non-negative variables is allowed to be positive. The target power during slot $k$ is given by $p_{\text {Target }, k} \in \mathbb{R}$. The objective can be linearized by writing the MILP in epigraph form.

In the case of cost optimization, the objective is the minimization of the sum of all energy costs in a given time horizon:

$$
\min _{p .} \sum_{k=0}^{K} \Delta_{k} \cdot\left(c_{\text {Draw }, k} \cdot p_{\text {Draw }, k}-c_{\text {FeedIn }, k} \cdot p_{\text {FeedIn }, k}+c_{\mathrm{Gas}, k} \cdot p_{\mathrm{Gas}, k}\right) .
$$

Here, power values are converted into energy by multiplying them with the slot length $\Delta_{k}=5 \mathrm{~min}$. Costs for electricity and gas per $\mathrm{kWh}$ in slot $k$ are denoted by $c_{\text {Draw }, k}$ and $c_{\text {Gas }, k}$. Electricity feed-in to the grid is compensated with $c_{\text {FeedIn, } k \text {. Gas is neglected for }}$ households without a CHP plant. 
All optimization models have either a CHP plant with a hot water tank, a BESS, or both. Input parameters are the electrical and thermal demands for either summer, winter, or the intermediate seasons, the energy tariffs, the initial state of charge (SoC) of the BESS, and the initial water tank temperature. The optimization variables comprise, aside from some auxiliary variables, the power supplied by the grid, the power feed-in, the BESS (dis-)charge power $p_{B, k}$, and binary variables that determine whether the CHP plant is running or not. When calculating the SoC of the BESS, the efficiency $\eta_{B}$ is used as follows:

$$
\mathrm{SoC}_{k+1}=\mathrm{SoC}_{k}+\Delta_{\mathrm{k}} \cdot\left\{\begin{array}{ll}
\eta_{B} \cdot p_{B, k} & , p_{B, k} \geq 0 \\
\frac{1}{\eta_{B}} \cdot p_{B, k} & , p_{B, k}<0
\end{array}\right. \text {. }
$$

Heat losses of the hot water tank $p_{\text {LossHwt,k }}$ are given by:

$$
p_{\mathrm{LossHwt}, \mathrm{k}}=a_{\mathrm{Hwt}} \cdot\left(12+5.93+v_{\mathrm{Hwt}}^{0.4}\right) \cdot \frac{\theta_{\mathrm{Hwt}, k}-\theta_{\mathrm{Env}}}{40 \mathrm{~K}}
$$

using the environmental temperature $\theta_{\mathrm{Env}}$, the hot water tank temperature $\theta_{\mathrm{Hwt}, k}$, and the volume of the tank $v_{\mathrm{Hwt}}$ in liters. The factor $a_{\mathrm{Hwt}}$ is set to 1 and hence the heat loss at $\theta_{\mathrm{Hwt}, k}=60^{\circ} \mathrm{C}$ is equal to the boundary between the European space heater efficiency classes B and C (European Union 2013). To add further constraints and reduce the number of feasible CHP load profiles, we assume that the plant has to keep its state of operation, i.e., being on or off, for at least $15 \mathrm{~min}$ which equals the length of 3 time slots. The BESS, on the contrary, is not restricted by such a constraint.

\section{Simulation models}

Aside from generating load profiles based on some criterion of optimality by using one of the MILPs, two simulation models are employed to explore further feasible and infeasible load profiles, which are far from being optimal in any sense. In both models, the DERs' behavior is determined by their internal state, defining how much electricity is consumed or produced and which actions are valid. Once a DER transitions into an infeasible state, e.g., by violating a storage restriction or by keeping a state for too short, a load profile is deemed infeasible. The SoC of the BESS and the thermal losses of the tank are modeled as given in Eqs. 3 and (4). While the BESS may change its operation mode at any time, the CHP plant has to remain in one mode for at least $15 \mathrm{~min}$. In the simulation, as opposed to the optimization, mode changes may occur at any time and thus are not restricted to the beginning of each $5 \mathrm{~min}$ time slot.

\section{Simulation model 1: random behavior}

In this simulation model, which is used to generate training and evaluation data for the patterns $\boldsymbol{A}$ and $\boldsymbol{D}$, the DERs operate on their own by making random operational choices. To generate feasible load profiles, possible choices may be restricted to those deemed valid at the current time. However, even though the simulation intends to produce a feasible profile, due to neglecting the future, situations may arise in which only invalid choices are left and thus the resulting load profile is infeasible. In order to improve the classification, we also generate infeasible profiles. Since all decisions are made randomly, simply allowing invalid choices does not necessarily result in an infeasible profile. Overall, a balance between the probabilities of valid and invalid choices has to be determined to generate infeasible load profiles that are similar to feasible ones. The simulations of either the BESS 
or the CHP plant lead to clear statements about the feasibility of the resulting load profiles. The simulated and aggregated load of the combination of both, on the contrary, may be incorrectly classified as infeasible. For example, in a simulation run, the BESS could make the invalid choice of exceeding its capacity of $1 \mathrm{kWh}$ by continuously providing $1 \mathrm{~kW}$ for more than an hour and thereby invalidate the feasibility of the aggregated profile. The same aggregate, on the other hand, could also be achieved by simply activating the CHP plant and thus may still be feasible. We deal with this issue by evaluating all infeasible schedules of this particular configuration with the MILP introduced above according to the rules derived later in this section.

\section{Simulation model 2: control-vector-based behavior}

The purpose of this simulation model is the generation of load profiles for pattern $C$. In this model, the DERs receive a control vector, defining the actions they have to perform in a certain time slot. Essentially, this input vector is an abstract representation of the resulting load profile. Since the overall feasibility can not be guaranteed for an arbitrary control sequence, either the input has to be classified according to its feasibility or a similar but feasible and thus repaired sequence has to be found. We repair the input vector by making iterative adaptions, changing only elements related to time steps up to the time the DER state becomes infeasible. For our tests, we studied two different representations:

1. A control sequence determining the operational mode of the DER for one day in 15 min intervals.

2. Six $4 \mathrm{~h}$ time slots in which one mode is activated once for a given time. The representation is the mode and the associated time for every time slot.

The first representation is very similar to an actual load profile and hence closely related to pattern $\boldsymbol{D}$. However, the major difference to pattern $\boldsymbol{D}$ is that, here, the repair is achieved using a set of simple rules instead of solving an optimization problem. The second representation is considered in order to test a more abstract approach. Both representations are first attempts and there are many other possibilities to represent load profiles.

\section{Evaluation of load profile feasibility}

While load profiles found by one of the optimization models are definitely feasible for the particular household configuration, feasibility is not known for randomly generated load profiles. Furthermore, a load profile generated by a simulation with multiple DERs may mistakenly be classified as infeasible. Therefore, these profiles are used as targets and validated by solving the corresponding MILP with the objective given in Eq. 1. Optimally, a feasible profile would lead to a target function value of zero. Unfortunately, this is usually not the case. In contrast to the simulation models, the optimization models are able to change the mode of operation only at the beginning of a time slot, i.e., every $5 \mathrm{~min}$. Additionally, the solver may prematurely stop the optimization once the optimality gap, i.e., the difference between the best found solution and a lower bound, is below a certain threshold or a given time limit is exceeded. For this reason, it is very unlikely that the target profile is matched exactly. To deal with this issue, we define feasibility and infeasibility thresholds for both the objective value $z$, i.e., the maximum absolute deviation, and the mean slot-wise absolute difference $\bar{d}$ between the target and the solution. The exact values, which are justified below, can be found in Table 4 . 
Table 4 Thresholds used in load profile evaluation

\begin{tabular}{llll}
\hline Configuration & Value & Feasible $(<)$ & Infeasible $(\geq)$ \\
\hline BESS & $z$ & $89 \mathrm{~W}$ & $89 \mathrm{~W}$ \\
& $\bar{d}$ & $89 \mathrm{~W}$ & $89 \mathrm{~W}$ \\
$\mathrm{CHP}$ & $z$ & $1050 \mathrm{~W}$ & $1050 \mathrm{~W}$ \\
& $\bar{d}$ & $44 \mathrm{~W}$ & $350 \mathrm{~W}$ \\
BESS and CHP & $z$ & $1139 \mathrm{~W}$ & $1139 \mathrm{~W}$ \\
& $\bar{d}$ & $133 \mathrm{~W}$ & $439 \mathrm{~W}$ \\
\hline
\end{tabular}

z: maximum absolute deviation, $\bar{d}$ : average absolute deviation

Profiles that have values of $z$ and $\bar{d}$ below the feasibility thresholds are deemed feasible. If at least one of both values is greater than or equal to the respective infeasibility threshold, the load profile is guaranteed to be infeasible. Although there might be indistinguishable load profiles, none occurred in our data.

As opposed to its MILP formulation, the simulated BESS is able to change its mode multiple times within a single time slot. The target power for this slot, which is passed to the optimization, is the average power. Since the optimization solver can decide only once per slot and based on the target power, simply choosing power $p_{B, k}$ equal to the target power $p_{\text {Target, } k}$ for a given slot $k$ may not be valid. Based on (3) and since we assumed a constant efficiency $\eta_{B}<1$, choosing $p_{B, k}=p_{\text {Target, } k}$ is only problematic when the original load profile requires charging and discharging within the same time slot. In this case, the resulting SoC is lower than the average power suggests ${ }^{1}$. Depending on the original load profile, the optimized power may have to reproduce the actual SoC by deviating from the target. With regard to the given model parameters, i.e., a (scaled) BESS with a power between $-1 \mathrm{~kW}$ and $1 \mathrm{~kW}$, this deviation is at most:

$$
-\frac{\Delta_{k}}{2} \cdot\left(\eta_{B} \cdot 1 \mathrm{~kW}-\frac{1}{\eta_{B}} \cdot 1 \mathrm{~kW}\right) \approx 7 \mathrm{Wh}
$$

This deviation is equal to a 5 min load of $84 \mathrm{~W}$. Adding a margin equal to the optimality gap of $5 \%$ and rounding up leads to the threshold of $89 \mathrm{~W}$ for $z$ and $\bar{d}$ (cf. configuration "BESS" in Table 4).

The CHP plant may be either running and generating a (scaled) electrical power of $1 \mathrm{~kW}$ or idling at $0 \mathrm{~kW}$. In a feasible $\mathrm{CHP}$ load profile, the operation mode can only change once within 3 consecutive slots, i.e., every $15 \mathrm{~min}$. Further restrictions arise from the temperature constraints of the hot water tank. In the worst case, the tank restrictions prevent setting the correct CHP mode, thereby leading to a deviation of less than $1000 \mathrm{~W}$. Adding a margin of $5 \%$, the value of $z$ is set to $1050 \mathrm{~W}$ (cf. configuration "CHP" in Table 4). Assuming the deviation happens every time the mode is changed, which is at most in 96 of the 288 slots, the average absolute deviation can not exceed $333 \mathrm{~W}\left(\approx 1000 \mathrm{~W} \cdot \frac{96}{288}\right)$. Considering the gap, we end up using a threshold for $\bar{d}$ of $350 \mathrm{~W}$. The feasibility threshold for the average absolute deviation is set to $44 \mathrm{~W}\left(\approx 1050 \mathrm{~W} \cdot \frac{12}{288}\right)$, which is reached when there are 12 mode changes with a deviation of $1050 \mathrm{~W}$ each. The number of 12 mode changes is the maximum number of mode changes observed in the simulated load profiles. For the household configuration with both BESS and CHP all values are added up. 


\section{ANN design}

For each of the usage patterns described in the approach section, the ANN has to represent a specific aspect of the abstracted energy flexibility. These aspects are learnt from examples generated by the models that have been described in the previous sections. We compare instances of the following classes of artificial neural network topologies by how well they are able to internalize the characteristics of the specific models:

(FC) Feed-forward neural networks with fully-connected layers are evaluated as a baseline.

(CNN) Convolutional Neural Networks are evaluated based on the idea that these networks should detect or generate recurring patterns in the load profile.

(RNN) Finally, we explore Recurrent Neural Networks motivated by the idea that the network should retrace a load profile step-by-step in temporal order, performing a time-discrete simulation of the physical system. We use long short-term memory units (Hochreiter and Schmidhuber 1997) as recurrent units.

Our main goal is to analyze overall practicability of the proposed approach. That is why we use only a small amount of preferably simple topologies of each class in experiments and do not systematically tune the hyper-parameters of the neural networks. The input data, models, logs, and results are tracked with Sacred 0.7.2 and published on GitHub (see declaration on data availability for a link). We used Keras 2.1.1 with the TensorFlow 1.4.1 backend to model and train our ANNs.

Figure 3 depicts the evaluated neural networks used for the feasibility classification task and Fig. 4 shows the neural networks used for the load generation/prediction tasks. Each neural network layer is represented by rounded rectangles and the rectangles represent the input and output vectors. The first number in the layer is the number of neurons or units. The abbreviation "FC" stands for fully-connected layer, "ReLU" is a rectangular linear unit, and "LSTM" is a Long/Short-Term Memory unit (Hochreiter and Schmidhuber 1997). During Up-Sampling, each input vector element is repeated several times.

\section{Training and evaluation methodology}

The data sets for each pattern and each combination of DER are pre-generated, shuffled and split into $80 \%$ used for training and validation and $20 \%$ used for the model evaluation presented in the results section. For the training of the ANNs, these $80 \%$ are split again into training and validation sets using a ratio of 90/10. In all cases, we use the Adam optimizer with the default parameters recommended in (Kingma and Ba 2014). The best model of each ANN is selected based on the lowest loss value for the validation data after each epoch. This model is evaluated using the $20 \%$ test data which remains unused for model training/validation. We train all networks with a batch size of 1024 and up to 5000 epochs. The training is stopped whenever the validation loss does not improve in the last 100 epochs (early stopping with 100 epochs patience). Regularization of weights or application of dropout is usually applied to achieve better generalization (Goodfellow et al. 2016). We only use dropout in our classification networks.

\section{DER state representation}

The input of the network is a concatenation of a vector representing the current state of the DER and the environment, namely the season, as well as a pattern-specific vector. 


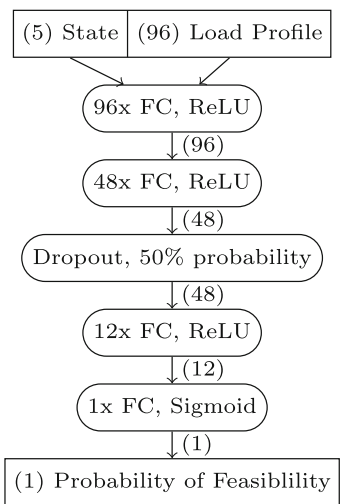

a

(5) State (96) Load Profile

$$
\downarrow(96)
$$

$48 \times 1 \mathrm{D}$-Convolution

kernel size 5 , padding 2 , ReLU

$\downarrow(96 \times 48)$

Max Pooling, size 2

$\downarrow(48 \times 48)$

$48 \times 1$ D-Convolution

kernel size 3 , padding 1 , ReLU

$\downarrow(24 \times 48)$

Max Pooling, size 2

$\downarrow(24 \times 24)$

$24 \times 1 \mathrm{D}-$ Convolution,
kernel size 3 , padding $1, \operatorname{ReLU}$

$\downarrow(12 \times 24)$

Max Pooling, size 2

$\downarrow(12 \times 24)$

Dropout, $50 \%$ probability

(5) $\underset{12 \times \text { FC, ReLU }}{\downarrow}$

$\downarrow(12)$

1x FC, Sigmoid

$\downarrow(1)$

(1) Probability of Feasiblility

b

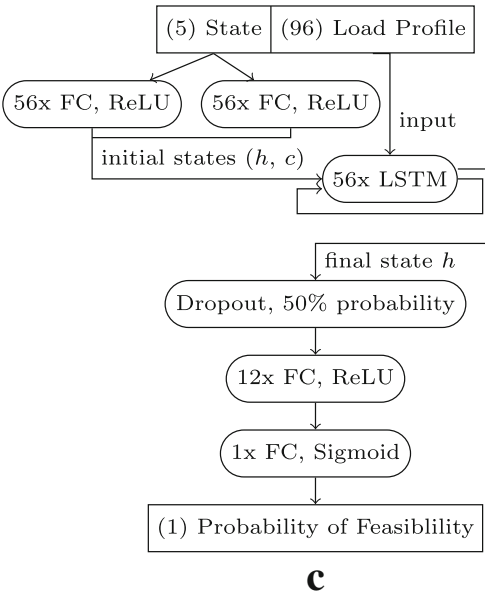

c

Fig. 3 Neural Network Topologies for Classification. a FC network, b CNN, c RNN

In detail, the state consists of a one-hot-encoded season vector (winter, intermediate, or summer) followed by the state of charge of the CHP plant's hot water tank and the state of charge of the BESS. If the modeled setup does not contain a CHP plant or BESS, the particular SoC is always set to zero.

For the CNNs and RNNs, applying the convolution or the recurrent time steps on the DER state does not seem plausible. Instead, our classification CNN processes the DER state in layers after the convolution (e.g., see Fig. 3a). The CNNs for generating load profiles process the DER state in the first fully-connected layer (e.g., see Fig. 3b). The RNN converts the DER state into initial states of the memory units (e.g., see Fig. 3c).

\section{Pattern A: load profile classification}

The ANN for load profile classification has to perform a binary classification task based on a DER state and a $24 \mathrm{~h}$ load profile divided into 96 time slots of $15 \mathrm{~min}$. The output of 

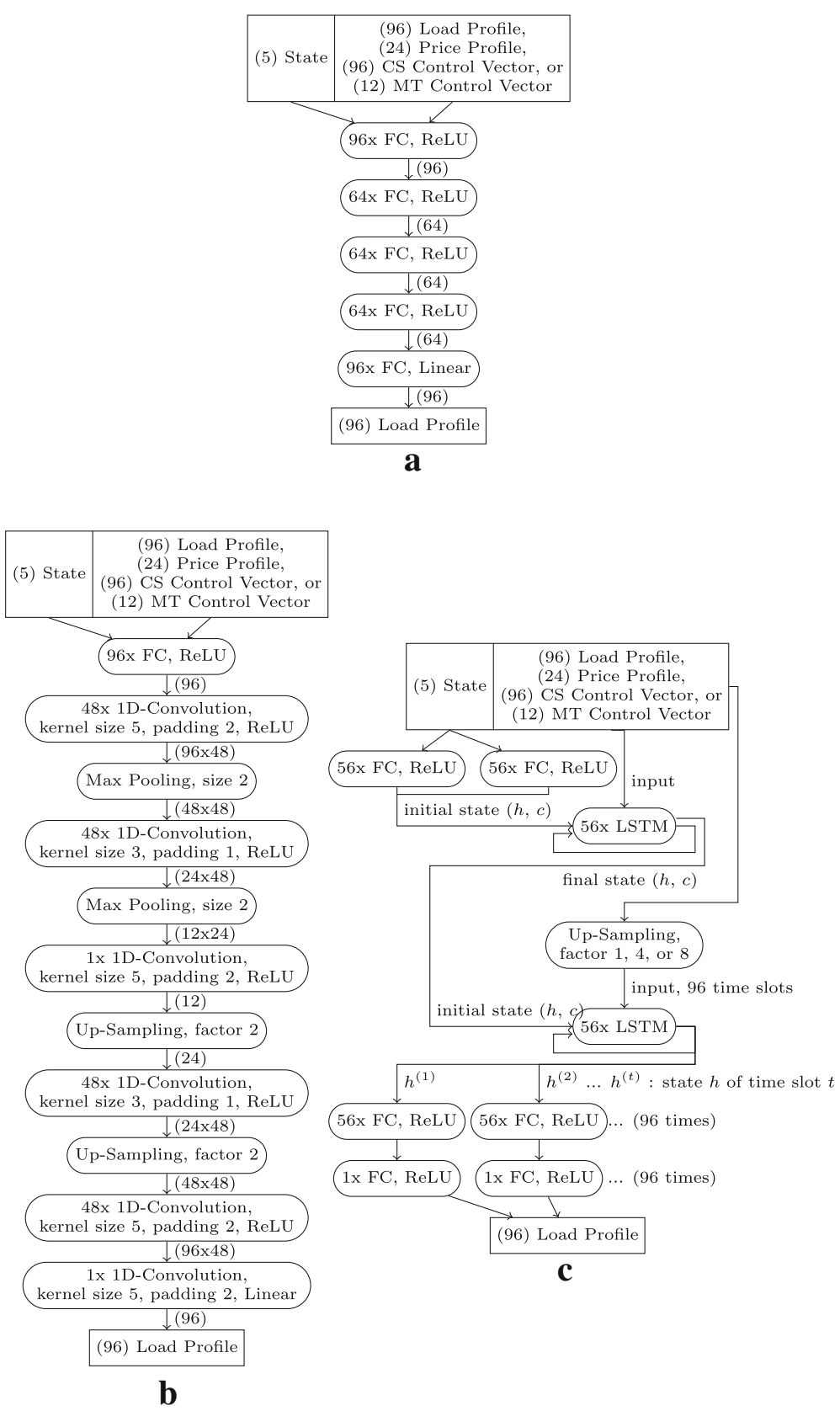

Fig. 4 Neural Network Topologies for Load Profile Prediction / Repair. a FC network, b CNN, c RNN

the network represents the probability (or belief) $P$ (feasible) that the given load profile is feasible for the DER in the given state. We use a sigmoid activation function in the output layer and binary cross-entropy as loss function, which is the recommended default for binary classification tasks (Goodfellow et al. 2016). We classify a load profile as feasible whenever:

$P($ feasible $)>P($ infeasible $) \Leftrightarrow P($ feasible $)>0.5$. 
The best performing ANN architectures within our experiments for each topology class are depicted in Fig. 3. To be able to compare the topology classes, the architectures are designed so that each topology has approximately 15,000 trainable parameters.

\section{Pattern B: price-based load profile forecasting}

In this pattern, the ANN predicts the load profile of the DER for a given price profile and state. We expect the ANNs to generate a $24 \mathrm{~h}$ load profile divided into $15 \mathrm{~min}$ time slots. Therefore, there are 96 output neurons with linear activation. The loss function used for training is the mean squared error. The expected load profiles are scaled such that the numerical representations remain within the interval $[-1,1]$.

The ANN topologies for patterns $\boldsymbol{B}$ to $\boldsymbol{D}$ are created based on the classification topologies, where the input data dimensionality is gradually reduced in each layer. The load profile generating ANNs, also, reduce the input data dimensionality to a certain degree, but then increase the dimensionality until the desired number of time slots for the load profile is reached. This way, these topologies resemble autoencoders (cf. (Goodfellow et al. 2016)). The resulting topologies are shown in Fig. 4. To be able to compare the three topology classes, the architectures for load prediction are designed such that the number of trainable parameters is approximately 30,000 .

\section{Pattern C: load profile generation}

In this pattern, the task of the model is to generate a load profile based on a control vector for the DER. Again, the load profiles are scaled to not exceed $\pm 1 \mathrm{~kW}$. The data for this pattern is generated using the control-vector-based model described above. The control sequences for the BESS and the CHP plant are divided into 96 slots of 15 min respectively. In addition, we evaluate a second control vector type, where a day is divided into six time slots of $4 \mathrm{~h}$. Each slot contains an operation mode and a duration describing how long the operation mode is active.

Except for the input dimensions of the first layer and an up-sampling factor, the ANN topologies used for this task are essentially the same as those for pattern $\boldsymbol{B}$. First experiments have shown that the performance of the RNN benefits from processing the control vector twice.

\section{Pattern D: load profile repair}

In this pattern, the $\mathrm{ANN}$ receives an infeasible load profile and has to construct a feasible one. Feasible profiles would be filtered beforehand using the classification pattern (i.e., an additional ANN). For our experiments we filtered according to the rules derived earlier in this paper. Both input and output are $24 \mathrm{~h}$ load profiles in time slots of $15 \mathrm{~min}$.

We evaluate the same ANN topologies used in patterns $\boldsymbol{B}$ and $\boldsymbol{C}$ and all load profiles are scaled to not exceed $\pm 1 \mathrm{~kW}$.

\section{Results and evaluation}

The practicality of our overall proposed approach depends heavily on the capability of ANNs to act as surrogate models for the flexibility of DERs. The ANNs must have a high classification accuracy and a low prediction error. We evaluated several ANNs for the patterns $A, B, C$, and $D$ and discuss the results in the following subsections. We also used the best CHP load profile classifier to validate whether real-world CHP load profiles are identified as feasible. 


\section{Load profile classification}

To measure the performance, we use the $F_{1}$-score and accuracy (Goodfellow et al. 2016). The $F_{1}$-score in terms of load profile feasibility is computed from precision, i.e., the share of positively classified profiles that are really feasible, and recall, i.e., the share of all feasible profiles that are positively classified, as follows:

$$
F_{1}=2 \cdot \text { precision } \cdot \text { recall } /(\text { precision }+ \text { recall }) .
$$

The evaluation results for load profile classification (pattern $\boldsymbol{A}$ ) are depicted in Fig. 5a. The proposed models achieve high $F_{1}$-scores indicating that a neural network can distinguish feasible and infeasible load profiles quite well. In our experiments, however, there was no single best topology for every DER setup. The RNN outperformed the other topologies for the setup with a BESS and the combination of BESS and CHP, whereas the $\mathrm{CNN}$ classified CHP load profiles slightly better. Looking at the average scores for each of the three setups, CHP load profiles were distinguished most accurately, whereas classifying the load profiles of the BESS/CHP combination was least accurate. One explanation for this result could be the fact that the CHP plant has the strongest operational restrictions and the BESS/CHP combination has the most degrees of freedom for its operation. The precisions of positive and negative choices for the classification of load profiles of a single CHP plant are similar to those achieved by Neugebauer et al. in (2017).

Preliminary experiments to classify feasibility based on $5 \mathrm{~min}$ averages instead of $15 \mathrm{~min}$ averages did not improve the general results. We also tested training three independent ANNs, one for each season, instead of encoding the level of thermal consumption in the ANN input. Based on the given input data, there was no general improvement from using three separate ANNs for winter, summer and intermediate seasons. Therefore, we did not pursue this approach any further.

\section{Price-based load profile forecasting}

The evaluation results are shown in Fig. 5b. As a reference, the average load observed in the training data is determined, which is only one value. The predictor labeled "Const" in Fig. $5 \mathrm{~b}$ predicts just this average for every time slot. The best results were achieved by a fully-connected network topology.

As can be seen from the mean absolute error (MAE), the predicted load profiles are off by less than $5 \mathrm{~W}$ on average, i.e., the absolute error is less than $0.5 \%$ of the scaled power of either the BESS or the CHP plant. We suspect that such good results are achieved by the neural networks that simply memorize a reference load profile for each price profile, as there are only 33 valid price profiles and a load profile may be optimal for multiple input states. In the application context, this behavior is acceptable if the structure of the tariff does not change. If other price profiles are used, the ANN probably needs to be updated.

\section{Load profile generation}

The results for the evaluation of ANNs for load profile generation based on control vectors are shown in Fig. 5c. Although the same three neural network topologies were used for the different control vectors, the performance is very diverse: With a root mean squared error (RMSE) of $5 \mathrm{~W}$, the RNN achieves the best result for modeling the BESS driven by a control sequence (CS), whereas the best result for a CHP plant driven by a control sequence is an RMSE of $250 \mathrm{~W}$. 
$\mathbf{a}$
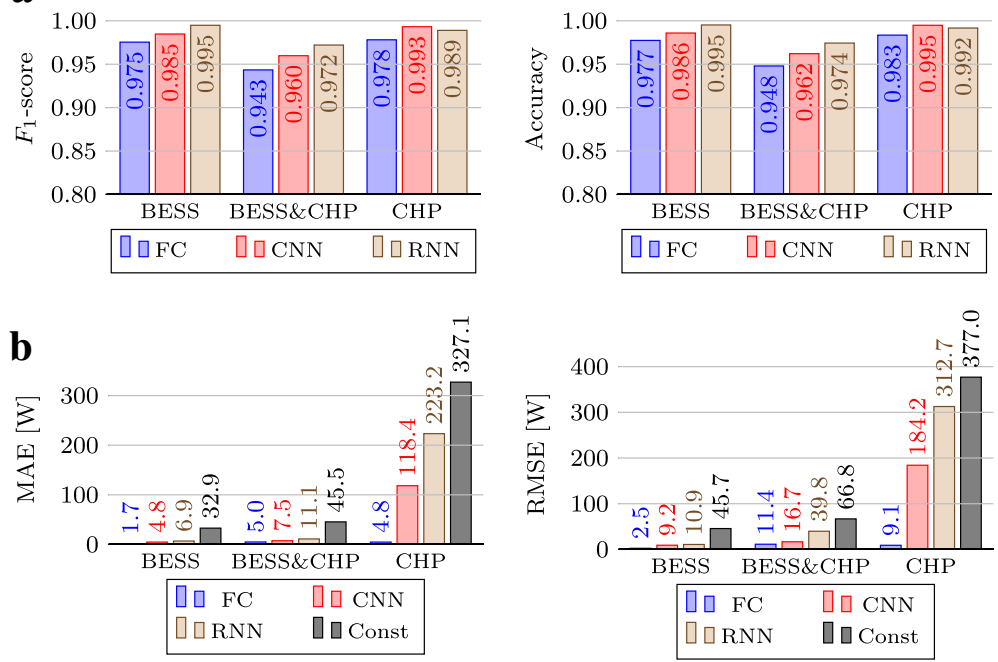

c
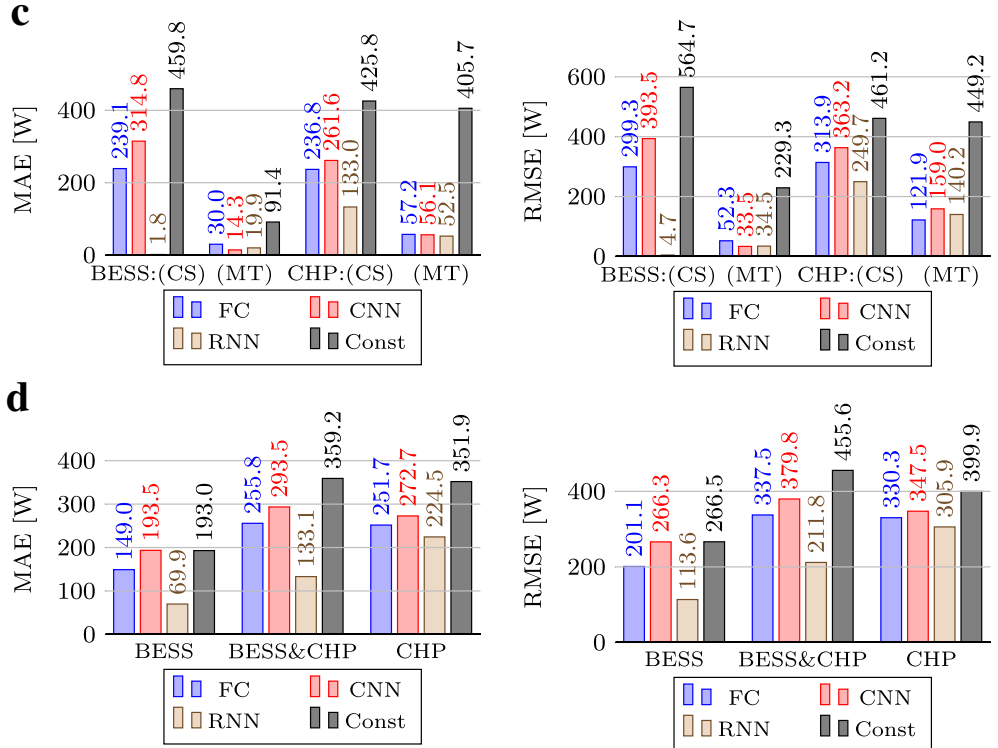

Fig. 5 Results for an ANN with a fully-connected feed-forward topology (FC), a convolutional neural network (CNN), and a recurrent neural network (RNN). a Classification Performance, b Load Prediction based on Price Profiles, c Load Generation based on Control Vectors, $\mathbf{d}$ Load Profile Repair (repairing only infeasible profiles)

In general, the models perform better for the battery storage than for the CHP plant. This may be caused by the activation of the plant due to thermal demand, especially for heating in the winter. The thermal demand is a latent variable which the ANN has to infer from the training samples. The BESS, on the other hand, is limited only by the energy storage capacity and the current state of charge.

\section{Load profile validation and repair}

The task assigned by the repair pattern seems to be the hardest for our neural models, as the results given in Fig. 5d show. Comparing our networks, the RNN has the lowest RMSE in all DER setups. But still, the mean absolute error of the RNN is above $70 \mathrm{~W}$. It is 
very likely that we did not find a suitable topology to solve the task of load profile repair. A fundamental problem may be posed by the fact that repairing an infeasible load profile can be done in many ways. Based on the optimization objective, there may be an infinite number of possible repaired load profiles having the same target function value. Hence, the profiles returned by the optimization are unlikely to follow a systematic scheme. In pattern $\boldsymbol{C}$, on the other hand, the control vector leads to a well-defined load profile. Based on the results of pattern $C$, we expect that ANNs are able to perform better on this task if the profiles are repaired in a consistent and simple way.

\section{Real-world CHP classification}

Finally, we tested the best-performing neural network that had been trained for classifying the feasibility of CHP load profiles (the $\mathrm{CNN}$ ) with real-world data. From a time series, we extracted 51 days from a period that is comparable to the summer state the ANN had been trained with. Based on the measured temperature of the hot water tank, 47 out of the 51 load profiles are correctly classified as feasible. A closer look at the four profiles that were classified as infeasible revealed that they originate from days with very little thermal demand. Hence, the CHP plant that generated these four profiles could not have satisfied the thermal consumption that is assumed in the model and thus the profiles were correctly classified as infeasible.

\section{Conclusion and outlook}

In this paper we evaluated the idea of using ANNs as surrogate models for the flexibility of DERs. A major advantage of this approach is that, for a demand side manager, there is no need to explicitly model DERs. In addition, the required amount of communication can be reduced to transmitting a few variables like DER states and prices or load profiles which is a significant advantage with respect to concerns about privacy or data economy.

As our results confirm, the concept of ANN-encoded abstracted flexibility is indeed viable and there are multiple ways of implementing it. We achieved the best results for the load profile classification, which we also successfully tested on real-world CHP data, and the price-based load profile forecasting patterns. While the mixed results from the load profile generation pattern indicate a general adequacy of the pattern itself, load profile repair did not work out well for training data generated by the optimization models. Since generation based on a control vector and repair are similar tasks, we conclude that this is likely due to the determinacy of the rules applied in the generation pattern, as opposed to the optimization which generates one of a possibly infinite number of repaired profiles. Hence, for future evaluations of the repair pattern there should be a well-defined solution.

As this paper presents first results of implementing the concept of ANN-encoded abstracted flexibility for energy management and smart grids, we are working on applying the concept in additional realistic scenarios, with more and other types of DERs, considering additional constraints, improving the used ANNs, as well as refining the patterns. Regarding the poor performance of our ANNs on some load profile generation tasks in contrast to the good classification performance, future research could take advantage of the classification ANN for a Generative Adversarial Network (Goodfellow et al. 2014) to generate more accurate load profiles. 


\title{
Endnote
}

${ }^{1}$ This may be deduced intuitively from $\eta_{B}<1$, (3) and:

$$
\begin{aligned}
& \text { Average Power: } p_{B, k} \quad=\frac{E_{B, C, k}-E_{B, D, k}}{\Delta_{k}} \\
& \text { Actual SoC: } \quad \operatorname{SoC}_{k+1}-\mathrm{SoC}_{k}=\eta_{B} \cdot E_{B, C, k}-\frac{1}{\eta_{B}} \cdot E_{B, D, k}
\end{aligned}
$$

with total amounts of energy charged $E_{B, C, k}$ and discharged $E_{B, D, k}$ during slot $k$.

\begin{abstract}
Abbreviations
ANN: Artificial neural network; BESS: Battery energy storage system; CHP: Combined heat and power; CNN: Convolutional neural network; CS: Control sequence; DER: Distributed energy resource; DR: Demand response; DSM: Demand side management; DSMgr: Demand side manager; EMS: Energy management system; FC: Fully-connected; LSTM: Long short term memory; MAE: Mean absolute error; MILP: Mixed integer linear program; ReLU: Rectified linear unit; RMSE: Root mean squared error; RNN: Recurrent neural network; SoC: State of charge; SVDD: Support vector data description
\end{abstract}

\section{Acknowledgements}

The authors would also like to thank the anonymous referees for their valuable reviews and helpful suggestions.

\section{Funding}

Publication costs for this article were sponsored by the Smart Energy Showcases - Digital Agenda for the Energy Transition (SINTEG) program. We gratefully acknowledge the financial support from the Federal in Ministry of Education and Research (BMBF) for the projects ENSURE (funding no. 03SFK1A) and KASTEL-SVI (funding no. 16KIS0521) and from the Federal in Ministry for Economic Affairs and Energy (BMWi) for the projects grid-control (funding no. 03ET7539G) and C/sells (funding no. 03SIN121).

\section{Availability of data and materials}

The data sets generated and used during the current study are available in the Github repository, https://github.com/ kfoerderer/ANN-Encoded-Abstract-Flexibility.

\section{About this supplement}

This article has been published as part of Energy Informatics Volume 1 Supplement 1, 2018: Proceedings of the 7th $\mathrm{DACH}+$ Conference on Energy Informatics. The full contents of the supplement are available online at https:// energyinformatics.springeropen.com/articles/supplements/volume-1-supplement-1.

\section{Authors' contributions}

KF proposed the concept, developed the usage patterns, implemented the simulation models and drafted major parts of the manuscript. MA provided and described the optimization model. KB helped developing the idea, and also designed, conducted and described the ANN implementations and experiments. IM assisted refining the usage patterns, and reviewed the drafts of the manuscript. HS provided feedback, supervision, organization of funding and resources for this collaboration. All of the authors have read and approved the final manuscript.

Competing interests

The authors declare that they have no competing interests.

\section{Publisher's Note}

Springer Nature remains neutral with regard to jurisdictional claims in published maps and institutional affiliations.

\section{Author details}

${ }^{1}$ FZI Research Center for Information Technology, Haid-und-Neu-Str. 10-14, 76131 Karlsruhe, Germany. ${ }^{2}$ Karlsruhe Institute of Technology, Institute AIFB, Kaiserstr. 12, 76131 Karlsruhe, Germany.

Published: 10 October 2018

\section{References}

Abuella M, Chowdhury B (2015) Solar power forecasting using artificial neural networks. In: 2015 North American Power Symposium (NAPS). IEEE. pp 1-5. https://doi.org/10.1109/NAPS.2015.7335176

Anders G, Schiendorfer A, Steghoefer JP, Reif W (2014) Robust scheduling in a self-organizing hierarchy of autonomous virtual power plants. In: ARCS 2014; 2014 Workshop Proceedings on Architecture of Computing Systems. IEEE. pp 1-8

Batra N, Kelly J, Parson O, Dutta H, Knottenbelt W, Rogers A, Singh A, Srivastava M (2014) Nilmtk: An open source toolkit for non-intrusive load monitoring. In: Proceedings of the 5th International Conference on Future Energy Systems. e-Energy '14. ACM, New York. pp 265-276. https://doi.org/10.1145/2602044.2602051

Bremer J, Lehnhoff S (2017) Hybrid Multi-ensemble Scheduling, vol. 10199(Squillero G, Sim K, eds.). Springer, Cham. https://doi.org/10.1007/978-3-319-55849-3_23

Bremer J, Rapp B, Sonnenschein M (2011) Encoding distributed search spaces for virtual power plants. In: 2011 IEEE Symposium on Computational Intelligence Applications In Smart Grid (CIASG). IEEE. pp 1-8. https://doi.org/10.1109/ CIASG.2011.5953329

Bremer J, Sonnenschein M (2013) Model-based integration of constrained search spaces into distributed planning of active power provision. Comput Sci Inf Syst 10(4):1823-1854 
Callaway DS, Hiskens IA (2011) Achieving controllability of electric loads. Proc IEEE 99(1):184-199. https://doi.org/10. 1109/JPROC.2010.2081652

European Union (2013) Commission Delegated Regulation(EU) No 812/2013 of 18 February 2013 supplementing Directive 2010/30/EU of the European Parliament and of the Council with regard to the energy labelling of water heaters, hot water storage tanks and packages of water heater and solar device Text with EEA relevance. Off J Eur Union 56(L 239):83-135

Faruqui A, George S (2005) Quantifying customer response to dynamic pricing. Electr J 18(4):53-63. https://doi.org/10. 1016/j.tej.2005.04.005

Faruqui A, Sergici S (2010) Household response to dynamic pricing of electricity: a survey of 15 experiments. J Regul Econ 38(2):193-225. https://doi.org/10.1007/s11149-010-9127-y

Förderer K, Ahrens M, Bao K, Mauser I, Schmeck H (2018) Towards the modeling of flexibility using artificial neural networks in energy management and smart grids. In: e-Energy '18. ACM, New York. https://doi.org/10.1145/3208903.3208915

Gellings CW (1985) The concept of demand-side management for electric utilities. Proc IEEE 73(10):1468-1470. https:// doi.org/10.1109/PROC.1985.13318

Goodfellow I, Bengio Y, Courville A (2016) Deep Learning. MIT Press, Cambridge. http://www.deeplearningbook.org

Goodfellow IJ, Pouget-Abadie J, Mirza M, Xu B, Warde-Farley D, Ozair S, Courville A, Bengio Y (2014) Generative Adversarial Networks. arXiv:1406.2661 [cs, stat]. arXiv: 1406.2661. Accessed 19 Jan 2018

Hinrichs C, Sonnenschein M (2017) A distributed combinatorial optimisation heuristic for the scheduling of energy resources represented by self-interested agents. Int J Bio-Inspired Comput 10(2):69-78. https://doi.org/10.1504/IJBIC. 2017.085895

Hochreiter S, Schmidhuber J (1997) Long Short-Term Memory. Neural Comput 9(8):1735-1780. https://doi.org/10.1162/ neco.1997.9.8.1735. Accessed 16 Jan 2018

Jargstorf J, Jonghe CD, Belmans R (2015) Assessing the reflectivity of residential grid tariffs for a user reaction through photovoltaics and battery storage. Sust Energ Grids Netw 1:85-98. https://doi.org/10.1016/j.segan.2015.01.003

Kingma DP, Ba J (2014) Adam: A Method for Stochastic Optimization. arXiv:1412.6980 [cs]. arXiv: 1412.6980. Accessed 15 Jan 2018

Kochanneck S, Schmeck H, Mauser I, Becker B (2015) Response of smart residential buildings with energy management systems to price deviations. In: 2015 IEEE Innovative Smart Grid Technologies - Asia (ISGT ASIA). IEEE

MacDougall P, Kosek AM, Bindner H, Deconinck G (2016) Applying machine learning techniques for forecasting flexibility of virtual power plants. In: 2016 IEEE Electrical Power and Energy Conference (EPEC). IEEE. pp 1-6. https://doi.org/10. 1109/EPEC.2016.7771738

Malbasa V, Zheng C, Chen PC, Popovic T, Kezunovic M (2017) Voltage stability prediction using active machine learning. IEEE Trans Smart Grid 8(6):3117-3124. https://doi.org/10.1109/TSG.2017.2693394

Mauser I, Müller J, Förderer K, Schmeck H (2017a) Definition, modeling, and communication of flexibility in smart buildings and smart grids. In: ETG-Fb. 155: International ETG Congress 2017. VDE Verlag, Berlin. pp 605-610

Mauser I, Müller J, Schmeck H (2017b) Utilizing flexibility of hybrid appliances in local multi-modal energy management. In: Proceedings of the 9th International Conference EEDAL'2017 - Energy Efficiency in Domestic Appliances and Lighting. Publications Office of the European Union, Luxembourg

McKenna E, Thomson M (2016) High-resolution stochastic integrated thermal-electrical domestic demand model. Appl Energy 165(Supplement C):445-461. https://doi.org/10.1016/j.apenergy.2015.12.089

Molderink A (2011) On the three-step control methodology for smart grids. PhD thesis. University of Twente. https://doi. org/10.3990/1.9789036531702

Neugebauer J, Bremer J, Hinrichs C, Kramer O, Sonnenschein M (2016) Generalized cascade classification model with customized transformation based ensembles. In: 2016 International Joint Conference on Neural Networks (IJCNN). pp 4056-4063. https://doi.org/10.1109/IJCNN.2016.7727727

Neugebauer J, Kramer O, Sonnenschein M (2015) Classification cascades of overlapping feature ensembles for energy time series data. In: Woon WL, Aung Z, Madnick S (eds). Data Analytics for Renewable Energy Integration. Springer, Cham. pp 76-93

Neugebauer J, Kramer O, Sonnenschein M, van den Herik J (2017) Instance Selection and Outlier Generation to Improve the Cascade Classifier Precision(Filipe J, ed.). Springer, Cham. https://doi.org/10.1007/978-3-319-53354-4_9

Nieße A, Sonnenschein M, Hinrichs C, Bremer J (2016) Local soft constraints in distributed energy scheduling. In: 2016 Federated Conference on Computer Science and Information Systems (FedCSIS). IEEE. pp 1517-1525

Palensky P, Dietrich D (2011) Demand side management: Demand response, intelligent energy systems, and smart loads. IEEE Trans Ind Inform 7(3):381-388

Rodrigues F, Cardeira C, Calado JMF (2014) The daily and hourly energy consumption and load forecasting using artificial neural network method: A case study using a set of 93 households in portugal. Energy Procedia 62:220-229. https:// doi.org/10.1016/j.egypro.2014.12.383. 6th International Conference on Sustainability in Energy and Buildings, SEB-14

Rohbogner G, Fey S, Benoit P, Wittwer C, Christ A (2014) Design of a multiagent-based voltage control system in peer-to-peer networks for smart grids. Energy Technol 2(1):107-120

Santo KGD, Santo SGD, Monaro RM, Saidel MA (2018) Active demand side management for households in smart grids using optimization and artificial intelligence. Measurement 115(Supplement C):152-161. https://doi.org/10.1016/j. measurement.2017.10.010

Severini M, Squartini S, Fagiani M, Piazza F (2015) Energy management with the support of dynamic pricing strategies in real micro-grid scenarios. In: 2015 International Joint Conference on Neural Networks (IJCNN). IEEE. pp 1-8. https:// doi.org/10.1109/IJCNN.2015.7280621

Toersche HA, Hurink JL, Konsman MJ (2015) Energy management with TRIANA on FPAI. In: 2015 IEEE PowerTech. IEEE. https://doi.org/10.1109/PTC.2015.7232650

Waffenschmidt E (2017) Cellular Power Grids for a 100 \% Renewable Energy Supply(Uyar TS, ed.). Springer, Cham. https:// doi.org/10.1007/978-3-319-45659-1_46 